\title{
Publisher Correction: miR-196b directly targets both HOXA9/MEIS1 oncogenes and FAS tumour suppressor in MLL-rearranged leukaemia
}

Zejuan Li, Hao Huang, Ping Chen, Miao He, Yuanyuan Li, Stephen Arnovitz, Xi Jiang, Chunjiang He, Elizabeth Hyjek, Jun Zhang, Zhiyu Zhang, Abdel Elkahloun, Donglin Cao, Chen Shen, Mark Wunderlich, Yungui Wang, Mary Beth Neilly, Jie Jin, Minjie Wei, Jun Lu, Peter J.M Valk, Ruud Delwel, Bob Lowenberg, Michelle M. Le Beau, James Vardiman, James C. Mulloy, Nancy J. Zeleznik-Le, Paul P. Liu, Jiwang Zhang \& Jianjun Chen

Nature Communications 3:688 doi: 10.1038/ncomms1681 (2012); Published 21 Feb 2012; Updated 10 Apr 2018

The original HTML version of this Article had an incorrect volume number of 2; it should have been 3 . This has now been corrected in the HTML; the PDF version of the Article was correct from the time of publication. 